experts and responders which produced qualitative and (4) quantative results development of competencies and testable objectives. The qualitative results showed that veterinarians and veterinary paraprofessionals require core competencies in all three groups and the four basic components of disaster management: mitigation, preparedness, response/emergency relief and recovery. A curriculum should cover all animals, companion, production and wild.

Prehosp Disaster Med 2011;26(Suppl. 1):s91-s92

doi:10.1017/S1049023X11003098

(A326) The Military Veterinarian's Role in Stabilization and Reconstruction Operations

J.C. Smith

Subsistence Directorate, Philadelphia, United States of America

Stabilization and reconstruction operations in failed or failing states require a bottom-up approach, focusing on the population as the strategic center of gravity. This bottom-up approach must address the population's basic needs as defined by Dr. Abraham Maslow's "Hierarchy of Needs" and provide a long-term means of self-sufficiency, rather than creating an "aid dependent economy”. Focusing stabilization and reconstruction operations on agricultural and agricultural related projects provides relief from donor dependency, stimulates economic growth, and thwarts the power of spoilers. Military veterinary personnel are uniquely qualified to design and implement agricultural stabilization and reconstruction programs in conjunction with the host-state ministries and agencies across the full range of military operations. Early, sustained engagement by military veterinarians stimulates agricultural productivity, improves animal and human health, directly supports the population's hierarchy of needs on all levels, and accelerates stabilization operations by reducing the population's susceptibility to spoilers.

Prehosp Disaster Med 2011;26(Suppl. 1):s92

doi:10.1017/S1049023X11003104

(A327) The Importance and Benefit of Disease and Injury Surveillance within Relief Operations

R.L. Burke

Geis Operations, Silver Spring, United States of America

Disease and injuries are expected consequences of disasters, either as direct result of the initial disaster or due to a collapse of the pre-existing public health infrastructure. While relief efforts are primarily directed at treating existing and preventing further disease and injury among victims of the disasters, it is also important to remain aware of the health impact on individuals and organizations providing assistance. The potential immune naïveness of relief workers may predispose them to contracting diseases which are normally not a concern for the local population. If significant numbers of relief workers are affected this can severely impact an organizations ability to provide assistance and may lead to a worsening of the situation. Even a simple surveillance program can provide early warning of potential problems in order to timely implement control measures which will prevent further illness and minimize mission impact.

Prehosp Disaster Med 2011;26(Suppl. 1):s92

doi:10.1017/S1049023X1100313X
(A328) Food and Water Risk Assessments during Disaster Operations

R.L. Burke

Geis Operations, Silver Spring, United States of America

Although the exact burden of foodborne disease is unknown, diarrheal diseases kill approximately 2.2 million people annually. Even in developed countries foodborne illness is estimated to affect over $20 \%$ of the population annually. During natural disasters existing food safety and security measures may be damaged and mission priorities during emergencies may prevent inspection agencies from conducting normal inspections and enforcing government regulations. This breakdown in the food safety infrastructure may lead to increases in foodborne diseases within the local population and relief workers. The risk in this latter group is possibly magnified by their immunologic naïveness to local pathogens and an outbreak among relief workers can severely impact support operations, interfere with the aid delivery, and may result in the loss of life. In addition to natural disease transmission, there is the potential for terrorist organizations to target relief workers through deliberate contamination of the food and water supplies. Consequently, relief agencies should consider both food safety and security during disaster operations. A Food and Water Risk Assessment (FWRA) is a tool for identifying potential high risk food items and practices in local food sources and facilities and examines the overall food operation, the food facilities and equipment, water potability, cleaning and sanitation, pest control, employee health and sanitation, food security, and the source of the food items. The FWRA identifies risk items and provides mitigative control measures designed to reduce the residual risk to acceptable levels and minimize potential disruption of mission operations. Although the ultimate goal is protecting the health of the relief workers, the FWRA can also be used as a tool to improve the food safety practices of local food facilities and suppliers which will in turn help to reduce the incidence of foodborne disease among the local population during the disaster relief operations and beyond.

Prehosp Disaster Med 2011;26(Suppl. 1):s92 doi:10.1017/S1049023X11003128

\section{(A330) Training Agricultural Emergency Responders}

\section{P.L. Cowen}

Veterinary Services, Professional Development Staff, Fort Collins, CO, United States of America

Training Agricultural Emergency Responders by Paula L. Cowen, D.V.M., Director, Professional Development Staff, Veterinary Services, Animal Plant Health Inspection Service, United States Department of Agriculture

Abstract: Background Emergency Response is a critical component of our Animal Agriculture infrastructure. The ability to deploy trained personnel to handle any kind of emergency is key to quickly containing any disaster and mitigating the effects. This training is provided by a number of federal agencies, universities as well as at the state and local level.

Body: Several training strategies are employed by a number of different entities. Training is available on-line, in the classroom, with wet labs using live animals, through exercises and case 